\title{
Single gene-based distinction of individual microbial genomes from a mixed population of microbial cells
}

\author{
Manu V. Tamminen * and Marko P. J. Virta \\ Department of Food and Environmental Sciences, University of Helsinki, Helsinki, Finland
}

Recent progress in environmental microbiology has revealed vast populations of microbes in any given habitat that cannot be detected by conventional culturing strategies. The use of sensitive genetic detection methods such as CARD-FISH and in situ PCR have been limited by the cell wall permeabilization requirement that cannot be performed similarly on all cell types without lysing some and leaving some nonpermeabilized. Furthermore, the detection of low copy targets such as genes present in single copies in the microbial genomes, has remained problematic. We

OPEN ACCESS

Edited by:

Stefan Bertilsson,

Uppsala University, Sweden

Reviewed by:

Claus Sternberg,

Technical University of Denmark,

Denmark

Brandon K. Swan,

Bigelow Laboratory for Ocean

Sciences, USA

*Correspondence:

Manu V. Tamminen,

Department of Food and Environmental Sciences, University of Helsinki, P.O. Box 56, 00014 Helsinki,

Finland

manu.tamminen@helsinki.fi

Specialty section: This article was submitted to Microbial Physiology and Metabolism, a section of the journal Frontiers in Microbiology

Received: 19 January 2015 Accepted: 23 February 2015 Published: 11 March 2015

Citation:

Tamminen MV and Virta MPJ (2015)

Single gene-based distinction of individual microbial genomes from a mixed population of microbial cells.

Front. Microbiol. 6:195.

doi: 10.3389/fmicb.2015.00195 describe an emulsion-based procedure to trap individual microbial cells into picolitervolume polyacrylamide droplets that provide a rigid support for genetic material and therefore allow complete degradation of cellular material to expose the individual genomes. The polyacrylamide droplets are subsequently converted into picoliter-scale reactors for genome amplification. The amplified genomes are labeled based on the presence of a target gene and differentiated from those that do not contain the gene by flow cytometry. Using the Escherichia coli strains XL1 and MC1061, which differ with respect to the presence $(X L 1)$, or absence (MC1061) of a single copy of a tetracycline resistance gene per genome, we demonstrate that $X L 1$ genomes present at $0.1 \%$ of MC1061 genomes can be differentiated using this method. Using a spiked sediment microbial sample, we demonstrate that the method is applicable to highly complex environmental microbial communities as a target gene-based screen for individual microbes. The method provides a novel tool for enumerating functional cell populations in complex microbial communities. We envision that the method could be optimized for fluorescence-activated cell sorting to enrich genetic material of interest from complex environmental samples.

Keywords: multiple displacement amplification, in situ PCR, fluorescent hybridization, flow cytometry

\section{Introduction}

The immense diversity of microbes in the environment is a serious obstacle to determine the presence of different genetic properties of individual microbial cells. Methods such as CARD-FISH and in situ-PCR that allow detection of low copy genetic targets in individual cells are limited by the different properties of bacterial cell surfaces that have to be permeabilized to allow diffusion of reagents into the cells without lysing them (Hodson et al., 1995; Kubota et al., 2008). Traditional FISH methods that rely on small oligonucleotide probes are less hampered by the permeabilization 


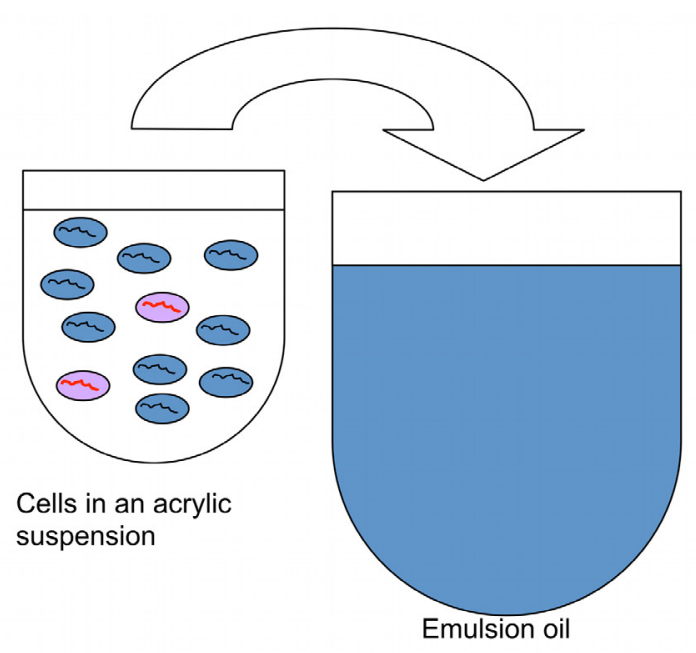

Mix with a magnetic stirrer

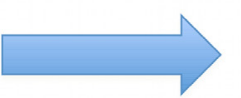

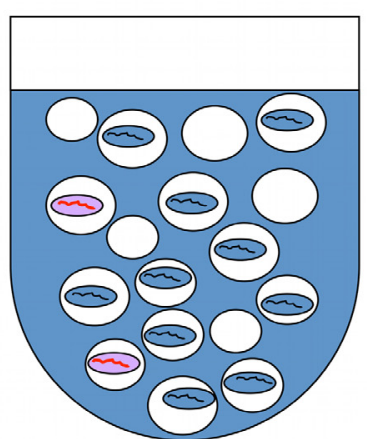

Acrylic emulsion in emulsion oil

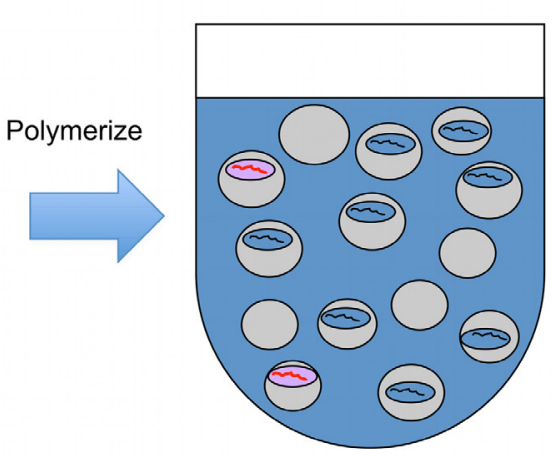

Polyacrylamide droplets in emulsion oil

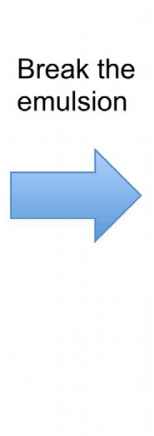

Polyacrylamide droplets with trapped cells

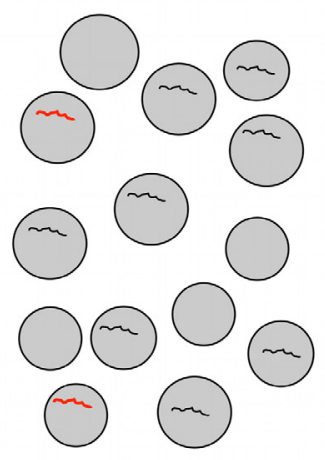

Polyacrylamide droplets with trapped genomes
FIGURE 1|A procedure to create polyacrylamide droplets containing individual genomes. Cells in acrylic suspension are mixed into emulsion oil. The emulsion droplets polymerize to yield polyacrylamide droplets containing single cells. The emulsion is broken and the cells in the polyacrylamide droplets are treated enzymatically to destroy cell walls, membranes, and protein components, and expose genomic DNA. Black lines represent genomes without the target gene, and red lines represent genomes with the target gene. requirements but on the other hand are limited to high copy targets such as ribosomal RNA (De Long et al., 1989; Amann et al., 1995). Combination of these two benefits to permit the detection of low copy genomic targets in diverse microbial populations has remained problematic.

Multiple displacement amplification (MDA) reaction has recently been developed for unspecific amplification of very low amounts of target DNA (Dean et al., 2002). The reaction has been shown to be capable of amplification of singlegenomes into microgram amounts of DNA (Marcy et al., 2007; Stepanauskas and Sieracki, 2007) and has also been shown to function in nanoliter reaction volumes (Marcy et al., 2007). Until now, the applicability of MDA to amplify rare genomic targets to assist FISH detection has not been demonstrated.

Here, we present a technique that permits an unbiased genome exposure of individual microbial cells by trapping them individually into rigid polyacrylamide droplets that support their genomes even after cell material has been degraded. The droplets are subsequently converted into picoliter-volume agarose reactors (picoreactors) and used for single-genome MDA reaction in an emulsion. A polymerase chain reaction (PCR)-based approach is used to fluorescently label the picoreactors containing genomic DNA that contains the target gene. Labeled and unlabeled picoreactors are differentiated using flow cytometry, which is capable of distinguishing target populations that constitute as little as $0.1 \%$ of the total microbial genome sample. In this proof-ofconcept study, we differentiate two Escherichia coli strains that differ with respect to the presence (E. coli XL1; Keasling et al., 1991) or absence (E. coli MC1061) of a singe copy per genome of a tetracycline resistance gene. We furthermore demonstrate the applicability of the technique to highly complex environmental samples by differentiating E. coli XL1 genomes from a mixture with diverse microbial cells extracted from marine sediment. The technique could be optimized for fluorescence-activated cell sorting to screen and capture genetic material of interest from complex environmental microbial communities. 


\section{Materials and Methods}

\section{Bacterial Immobilization in Polyacrylamide Beads}

Escherichia coli XL1 and MC1061 cells were cultivated in LB medium until reaching stationary phase, fixed for $2 \mathrm{~h}$ with $2 \%$ paraformaldehyde (Merck, Darmstadt, Germany) in PBS and stored in aqueous $50 \%$ ethanol at $-20^{\circ} \mathrm{C}$ until use. The cell density was determined using an LSR II flow cytometer (BectonDickinson, Franklin Lakes, NJ, USA). The microbial cells were extracted from the marine sediment using a previously published method (Kallmeyer et al., 2008) and fixed as described above. The cell density was determined using a Zeiss Axiovert $200 \mathrm{M}$ fluorescence microscope. The fixed cells were filtered through a $5-\mu \mathrm{m}$ nitrocellulose filter (Millipore, Cork, Ireland) and suspended in $250 \mu \mathrm{l}$ of acrylic suspension containing 20\% acrylamide (Sigma, St. Louis, MO, USA), $0.27 \%$ N,N'-bisacryloylcystamine (Sigma, St. Louis, MO, USA) and $0.5 \%$ ammonium persulphate (Sigma, St. Louis, MO, USA). The suspension was mixed as described in (Williams et al., 2006) by drop-wise addition using an ordinary pipette ( $5 \mathrm{~s}$ between drops) into $400 \mu \mathrm{l}$ of mineral oil containing 4.5\% Span 80 (Sigma-Aldrich, St. Louis, MO, USA), 0.4\% Tween 80 (Sigma, St. Louis, MO, USA) and 0.05\% Triton X100 (Applichem, Darmstadt, Germany) with stirring at 1,000 rpm using an IKA Yellowline MSH basic laboratory stirrer. After addition of the acrylic phase, TEMED (Sigma, St. Louis, MO, USA) was added on top of the emulsion to a concentration of $3.8 \%$ (25 $\mu \mathrm{l}$ of TEMED). Stirring was continued for $1 \mathrm{~h}$ to allow complete polymerization. After polymerization, the emulsion was disrupted with $2 \mathrm{ml}$ of water-saturated diethylether (Sigma, St. Louis, MO, USA). The polyacrylamide droplets were washed by suspending into $2 \mathrm{ml}$ of sterile water and recovered by centrifugation for $30 \mathrm{~s}$ at $8,000 \mathrm{~g}$. Washes were repeated until all traces of oil and ether had disappeared.

\section{Cell Lysis}

Polyacrylamide droplets $(200 \mu \mathrm{l})$ were suspended in $800 \mu \mathrm{l}$ of 1x PCR buffer (Fermentas), after which $13 \mathrm{U}$ of proteinase $\mathrm{K}$ (Sigma-Aldrich, St. Louis, MO, USA) was added and the suspension was incubated at $37^{\circ} \mathrm{C}$ for $1 \mathrm{~h}$. After heat-inactivating proteinase $\mathrm{K}$ at $90^{\circ} \mathrm{C}$ for $1 \mathrm{~min}, 20 \mu \mathrm{g}$ of lysozyme (Sigma, St. Louis, MO, USA) was added and the suspension was incubated at $37^{\circ} \mathrm{C}$ for $1 \mathrm{~h}$. Finally, $26 \mathrm{U}$ of proteinase $\mathrm{K}$ was added and the suspension was incubated for $5 \mathrm{~h}$ at $55^{\circ} \mathrm{C}$. Enzymes were inactivated by heating at $90^{\circ} \mathrm{C}$ for $5 \mathrm{~min}$.

\section{Forming Agarose Shells on Polyacrylamide Droplets}

A 20- $\mu$ l aliquot of polyacrylamide droplets, prepared as described above, was mixed with $200 \mu \mathrm{l}$ of agarose IV solution (Amersco, $\mathrm{OH}, \mathrm{USA}$ ) to yield a $1 \%$ agarose suspension. The agarose suspension was kept at $45^{\circ} \mathrm{C}$ to prevent solidifying. The temperatureequilibrated agarose suspension was mixed drop-wise (5 s between drops) into $1 \mathrm{ml}$ of warm $\left(50^{\circ} \mathrm{C}\right)$ emulsion oil using an ordinary pipette, then stirred at $1,000 \mathrm{rpm}$ for $5 \mathrm{~min}$ and mixed with $4 \mathrm{ml}$ of ice-cold emulsion oil. After holding on ice for $15 \mathrm{~min}$, the suspension was divided into 2-ml microcentrifuge

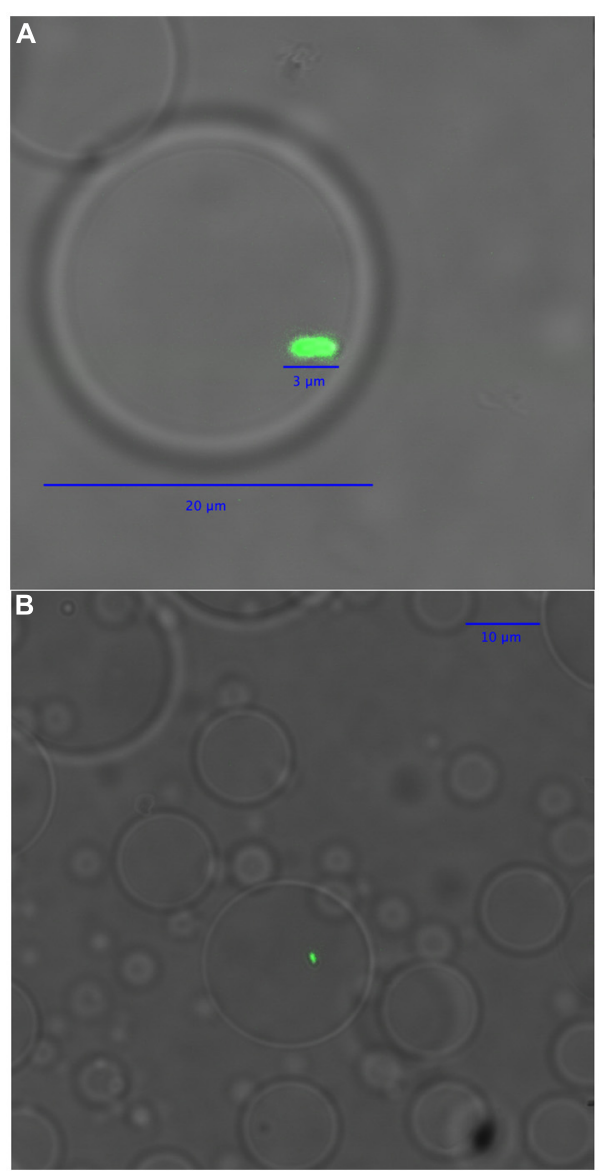

FIGURE 2 | A differential interference contrast/confocal micrograph of (A) a polyacrylamide droplet containing an Escherichia coli XL1 genome after cell lysis, and (B) several polyacrylamide droplets, one of which contains an $\boldsymbol{E}$. coli XL1 genome. The green fluorescence of SYBR Green dye is used to visualize DNA.

tubes (Eppendorf, Hamburg, Germany), and excess emulsion oil was removed by centrifuging for $10 \mathrm{~min}$ at $12,000 \mathrm{~g}$ and discarding the upper layer. The remaining emulsion oil was dissolved in water-saturated diethylether, and the picoreactors were washed as described above. Finally, agarose picoreactors were filtered through a cell strainer with a $40 \mu \mathrm{m}$ mesh (BD Falcon, Franklin Lakes, NJ, USA).

\section{MDA Reaction and Second Polyacrylamide Layer Formation}

To allow subsequent MDA reactions, the polyacrylamide matrix was dissolved by mixing $38 \mu \mathrm{l}$ of the agarose picoreactors in $150 \mu \mathrm{l}$ of a solution containing $87 \mu \mathrm{l}$ of Replig Buffer (Qiagen, Hilden, Germany), $10 \mu$ l of Repli-g Ф29 polymerase (Qiagen, Hilden, Germany), $1 \mu \mathrm{g} / \mu \mathrm{l}$ BSA (Roche, Mannheim, Germany) and 6.7 $\mu \mathrm{M}$ dithiothreitol (Qiagen, Hilden, Germany). The reagents, with the exception of the Repli-g Buffer, were sterilized under UV light for $10 \mathrm{~min}$ prior to use. The solution was mixed with $400 \mu$ l of emulsion oil 

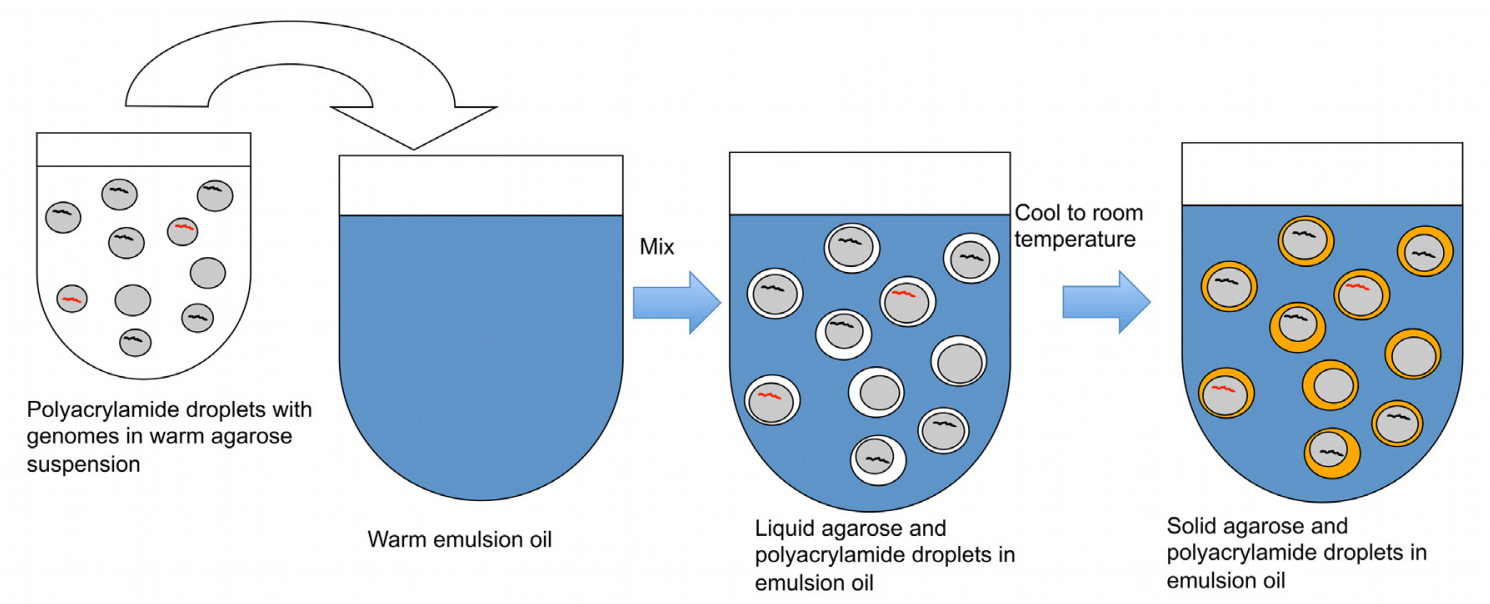

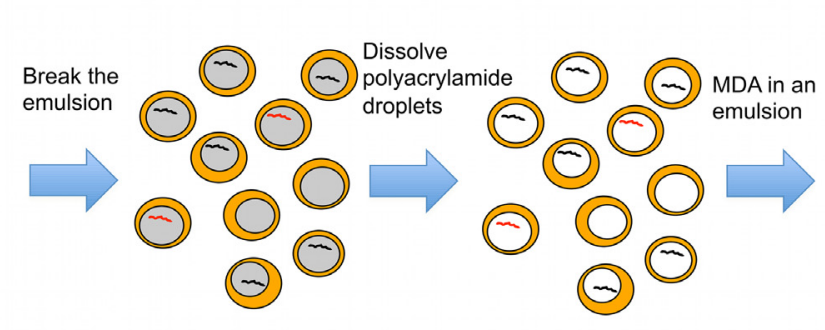

Agarose picoreactors before polyacrylamide dissolution
Agarose picoreactors

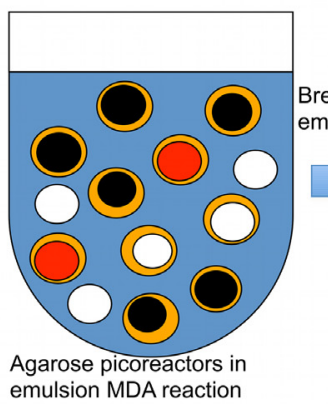
emulsion oil

FIGURE 3 | A procedure to create agarose picoreactors for single-genome amplification. An agarose layer is added onto polyacrylamide droplets that contain individual genomes (Figures 1 and 2). Multiple displacement amplification (MDA) is performed in an emulsion to ensure individual amplification of each genome. Black lines and black-filled circles represent target-less unamplified and amplified genomes, respectively. Red lines and red-filled circles represent target-containing unamplified and amplified genomes, respectively. White-filled circles represent empty picoreactors. and incubated overnight at $30^{\circ} \mathrm{C}$. The emulsion oil was dissolved in water-saturated diethylether, and the droplets were washed as described above. The agarose picoreactor droplets were suspended in an acrylic suspension prepared as above, but also containing an acrydite-modified forward primer ( $5^{\prime}$ Acrydite-TAC GTG AAT TTA TTG CTT CGG-3'; IDT, Berchem, Belgium) at a final concentration of $1.0 \mu \mathrm{M}$ in a total volume of $250 \mu \mathrm{l}$. The suspension was mixed drop-wise (5 s between drops) into emulsion oil while stirring at 1,000 rpm. After addition of the acrylic phase, TEMED was added on top of the emulsion to a concentration of 3.8\% (25 $\mu \mathrm{l}$ of TEMED). Stirring was continued for $1 \mathrm{~h}$ to allow complete polymerization. After polyacrylamide polymerization, the emulsion oil was dissolved in water-saturated diethylether, and the polyacrylamide-agarose picoreactors were washed as described above.

\section{Emulsion PCR}

Polyacrylamide-agarose picoreactors $(148 \mu \mathrm{l})$ were mixed into 1x Hot Start PCR Buffer containing $2.0 \mathrm{mM} \mathrm{MgCl}_{2}$ (Fermentas, St. Leon-Rot, Germany), $0.2 \mathrm{mM}$ dNTP mixture (0.2 $\mathrm{mM}$ each, dTTP substituted with dUTP), $0.01 \mu \mathrm{M}$ forward primer $\left(5^{\prime}\right.$ TAC GTG AAT TTA TTG CTT CGG-3'), $2.5 \mu \mathrm{M}$ reverse primer (5'-ATA CAG CAT CCA AAG CGC AC-3'; Oligomer,
Helsinki, Finland), and 50 U of Maxima Hot Start Taq Polymerase (Fermentas, St. Leon-Rot, Germany) in a total reaction volume of $200 \mu \mathrm{l}$. The PCR mixture was added drop-wise to the PCR emulsion oil as described in (Williams et al., 2006) with constant stirring $(1,000 \mathrm{rpm})$, and then allowed to continue mixing for an additional $5 \mathrm{~min}$. The mixture was then divided into 50 $\mu l$ aliquots on PCR strips, overlaid with $50 \mu \mathrm{l}$ of mineral oil and subjected to PCR thermal cycling. Cycling conditions were $5 \mathrm{~min}$ at $95^{\circ} \mathrm{C}, 25$ cycles of $1 \mathrm{~min}$ at $95^{\circ} \mathrm{C}, 1 \mathrm{~min}$ at $55^{\circ} \mathrm{C}$ and $2 \mathrm{~min}$ at $72^{\circ} \mathrm{C}$, with a final extension of $10 \mathrm{~min}$ at $72^{\circ} \mathrm{C}$. After $\mathrm{PCR}$, the emulsion was pooled in $2 \mathrm{ml}$ microcentrifuge tubes (Eppendorf, Hamburg, Germany), and the excess emulsion oil was removed by centrifuging for $10 \mathrm{~min}$ at $12,000 \mathrm{~g}$ and discarding the upper layer. The remaining emulsion oil was dissolved in water-saturated diethylether, and the droplets were washed as described above.

\section{Picoreactor Labeling}

After PCR, polyacrylamide-agarose picoreactors were mixed with $400 \mu \mathrm{l}$ of hybridization buffer containing $0.9 \mathrm{M} \mathrm{NaCl}$ (SigmaAldrich, St. Louis, MO, USA), 20 mM Tris- $\mathrm{HCl}$ pH 8 (SigmaAldrich, St. Louis, MO, USA), $0.02 \%$ sodium dodecyl sulfate (Sigma, St. Louis, MO, USA) and 20\% formamide (Applichem, Darmstadt, Germany). After adding a 5'-Cy5-labeled red 


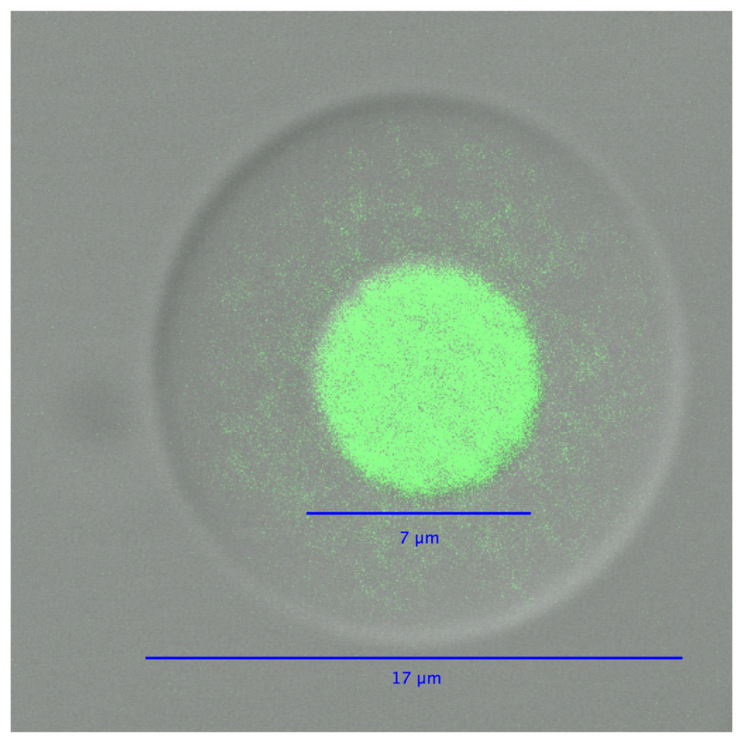

FIGURE 4 | A differential interference contrast/confocal micrograph of an agarose picoreactor after genome amplification in an emulsion MDA reaction. The green fluorescence of SYBR Green dye is used to visualize DNA.

fluorescent probe (5'-GCG CCT ATT AAT GAC AAC AA-3') to a concentration of $5 \mathrm{pM}$, the probe-picoreactor mixture was heated to $100^{\circ} \mathrm{C}$ for $2 \mathrm{~min}$ and then hybridized by incubating at $46^{\circ} \mathrm{C}$ for $1 \mathrm{~h}$. The hybridization mixture was washed by first incubating with hybridization buffer for $20 \mathrm{~min}$ at $48^{\circ} \mathrm{C}$ and then incubating with hybridization buffer without formamide for $20 \mathrm{~min}$ at $48^{\circ} \mathrm{C}$; thereafter, the washed picoreactors were suspended in $400 \mu \mathrm{l}$ of hybridization buffer without formamide. Between washing steps, picoreactors were collected by centrifuging mixtures for $30 \mathrm{~s}$ at $8,000 \mathrm{~g}$. A SYBR Green stock solution was added to a final concentration of $0.13 \%$ to give a green fluorescent signal to DNA-containing polyacrylamide-agarose picoreactors.

\section{Flow Cytometry and Microscopy}

The polyacrylamide-agarose picoreactors were analyzed based on green (fluorescein isothiocyanate, FITC) and red (allophycocyanin, APC) fluorescence using an LSR II flow cytometer (Becton-Dickinson, Franklin Lakes, NJ, USA). Successfully assembled picoreactors were separated from empty agarose droplets by increased side scatter value. The labeled picoreactors containing amplified E. coli XL1 genomic DNA (with the tetracycline resistance gene) could be differentiated from those containing amplified E. coli MC1061 genomic DNA (without the tetracycline resistance gene) by their increased fluorescence. Micrographs for were taken using SP5 (Figures 2A,B) and SP2 (Figure 4) confocal microscopes using objective HCX PL APO 63x/1.2W Corr, 0.17 CS (Leica, Wetzlar, Germany). The software used was LCS 2.61.1537 for SP2 and LAS AF 2.2.0 build 4765 for SP5. The differential interference contrast and confocal micrographs were combined, and the scales were drawn using ImageJ (Abramoff et al., 2004).

\section{Results}

\section{Bacterial Trapping in Polyacrylamide Droplets and Genome Exposure}

In the first part of the technique, acrylamide is polymerized on microbial cells, trapping cells in emulsion droplets (Figure 1). In this state, cell walls and other cellular components can be enzymatically removed, allowing microbial genomes to be exposed to further reactions. The exposed genomes do not diffuse into the surrounding liquid because they are covered and supported by the polyacrylamide matrix (Figures $\mathbf{1}$ and $\mathbf{2 A}$ ). The polyacrylamide is prepared using a special cross-linker that is dissolved by the mild reducing conditions of the subsequent MDA reaction.

When a dilute cell suspension is mixed into an emulsion, the number of cells in each emulsion droplet follows a Poisson distribution with an average number of cells per droplet of less than one. Empty emulsion droplets are most common, followed by droplets containing one cell; the frequency of droplets with more than one cell is very low compared with those with one cell. Therefore, mixing cells in an acrylamide suspension into an oil emulsion for polymerization is an efficient way to create polyacrylamide droplets that capture single cells. The diameter of the polyacrylamide droplets typically ranged from about 5-30 $\mu \mathrm{m}$ with most droplets having a diameter around $10 \mu \mathrm{m}$ (Figure 2B). The corresponding volume of the droplets therefore ranged from 0.07 to 14 picoliters, calculated using the formula $V=4 / 3 \pi r^{3}$.

\section{Creating Picoreactors and Performing Emulsion MDA}

In the second part of the technique, an agarose layer is added to the polyacrylamide droplets, which are subsequently dissolved prior to performing MDA reactions (Figure 3). The polyacrylamide must be dissolved because its polymer structure is too dense to allow an efficient MDA reaction. Dissolving the polyacrylamide droplet yields microbial genomes in picoliter liquid volumes within an agarose layer. These agarose picoreactors are mixed with reagents for the MDA reaction and then mixed into an emulsion. Agarose is permeable to enzymes and small molecules but not to genomic or amplified DNA (Figure 4). Because the only DNA in the agarose picoreactors comes from single-genomes trapped in the polyacrylamide droplets, the picoreactors provide a relatively sterile environment for the MDA reaction. The sterility of the reaction is also enhanced by performing the MDA reaction in an emulsion in which each picoreactor occupies an individual reaction compartment. Moreover, the contamination can be further decreased by UV treatment of the MDA reagents (Woyke et al., 2011).

\section{Labeling Picoreactors Containing Genomes with a Target Gene}

In the third part of the technique, a new layer of polyacrylamide containing immobilized PCR primers complementary to 
A
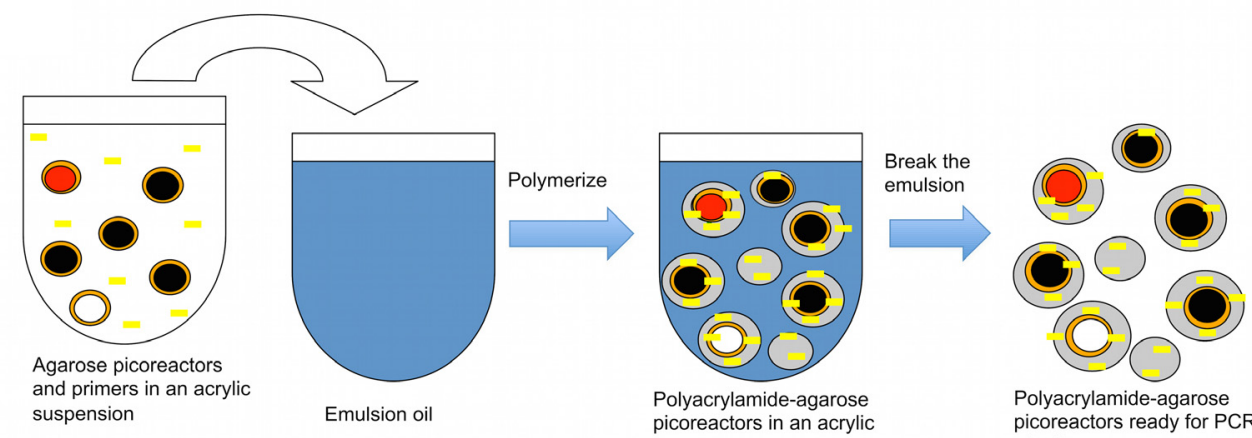

Polyacrylamide-agarose picoreactors in an acrylic suspension

Polyacrylamide-agarose picoreactors ready for PCR labelling

B

$$
\begin{aligned}
& \text { A target gene present in the } \\
& \text { picoreactor } \\
& \longrightarrow \text { Amplification }
\end{aligned}
$$

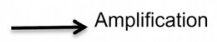

No target gene present in the picoreactor

$\longrightarrow$ No amplification
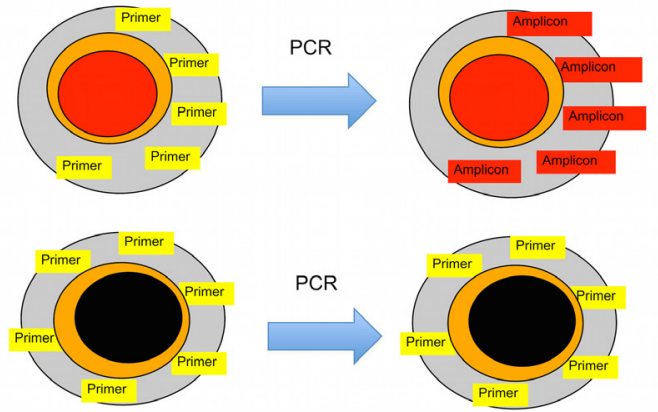

C

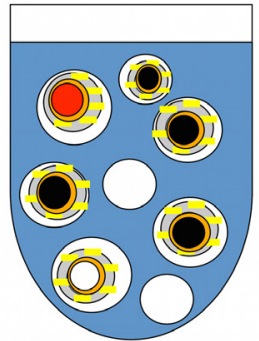

Polyacrylamide-agarose picoreactors before emulsion PCR
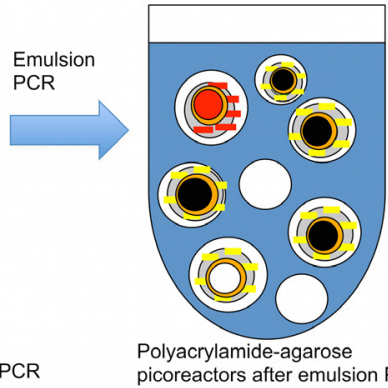

Polyacrylamide-agarose picoreactors after emulsion PCR

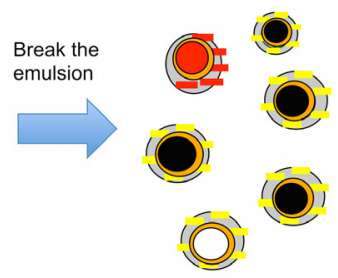

PCR-labelled polyacrylamideagarose picoreactors

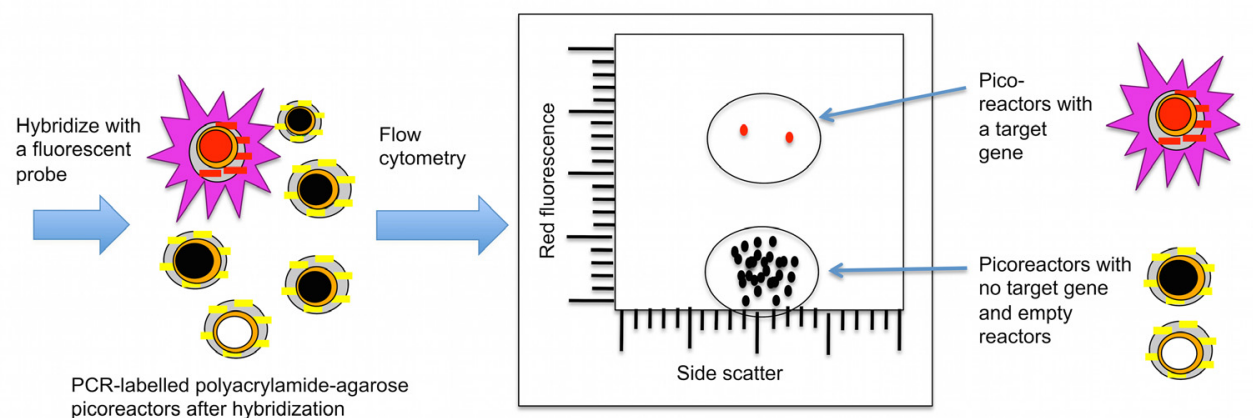

FIGURE 5|A layer of polyacrylamide is added to picoreactors to prepare them for PCR-based labeling. (A) Procedure for adding a polyacrylamide layer onto agarose picoreactors (prepared in Figure $\mathbf{3}$ ). The acrylamide suspension contains an acrydite-modified primer that becomes covalently attached to the polyacrylamide matrix during polymerization. (B) The polyacrylamide matrix contains a covalently attached primer complementary to the target gene. If the amplified genome in the picoreactor contains the target gene of interest (red-filled circle), an amplicon is synthesized by PCR that remains covalently attached to the polyacrylamide matrix. For droplets with no target gene of interest (black-filled circle, picoreactors containing genomes with no target gene; white-filled circle, empty picoreactors), no amplicon is generated by PCR. Agarose residues (orange) melt during PCR and do not interfere with the reaction. (C) After PCR, the droplets with attached amplicons are labeled using a complementary fluorescent probe. The labeled droplets are then differentiated by their increased fluorescence using a flow cytometer. 


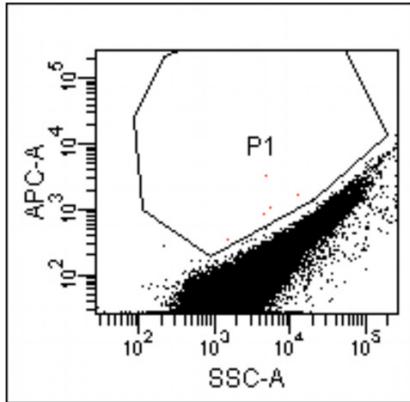

$0 \% \times L 1$ cells

5 events in $\mathrm{P} 1$

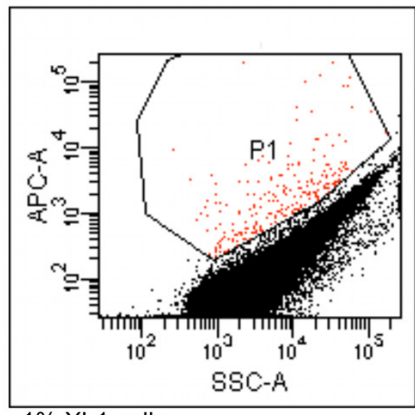

$1 \%$ XL1 cells

229 events in $\mathrm{P} 1$

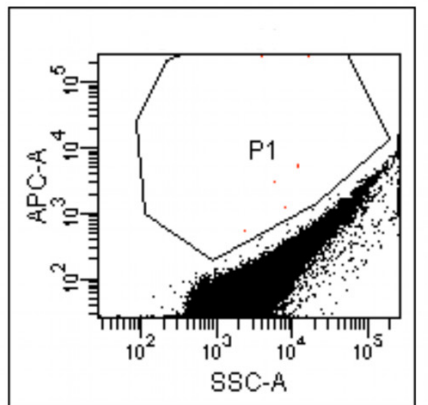

$0.01 \%$ XL1 cells

7 events in $\mathrm{P} 1$

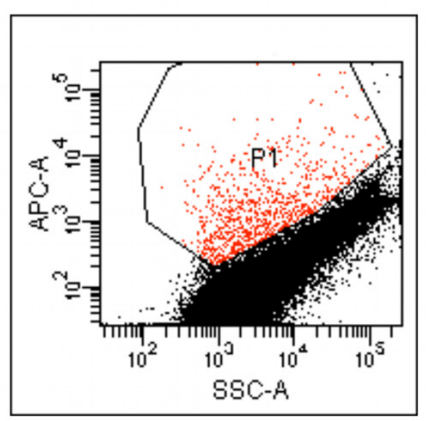

$10 \%$ XL1 cells

912 events in $\mathrm{P} 1$

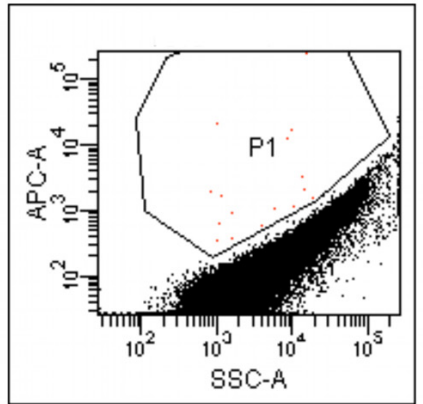

$0.1 \%$ XL1 cells

17 events in P1

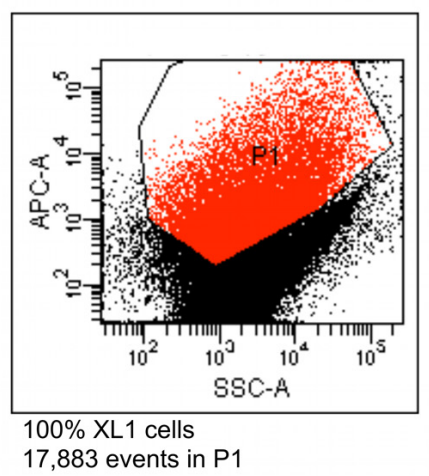

FIGURE 6 | Flow cytometric results from picoreactors of different suspensions of $E$. coli XL1 and E. coli MC1061. The E. coli XL1 genome contains a single copy of a tetracycline resistance gene, whereas the E. coli MC1061 genome contains none. The genomes are amplified in agarose picoreactors and labeled by emulsion PCR and fluorescent probe hybridization targeting the tetracycline resistance gene. Picoreactors containing the $\mathrm{XL1}$ genome exhibit increased red fluorescence. The parameter SSC-A on the $x$-axis refers to a side-scatter value that correlates with the light-scattering property of the analyzed particles. The parameter APC-A on the $y$-axes refers to the intensity of red fluorescence. Events in the P1 gate are labeled picoreactors containing XL1 genomes and therefore have increased red fluorescence. Altogether, 100,000 events were collected from each suspension. The fluorescent events in a suspension containing no XL1 cells $(0 \%)$ are false-positive events. The non-fluorescent events in suspensions containing 100\% XL1 cells represent empty picoreactors. the target gene is added to the picoreactors (Figure 5). The picoreactors are then mixed with PCR reagents to form an emulsion, and the target gene is amplified by PCR. PCR can be performed on the picoreactors because the new layer of polyacrylamide provides a matrix that supports the DNA generated by the MDA reaction and structurally reinforces the picoreactors, conferring heat-tolerance to them. The agarose in the picoreactors melts and does not interfere with the PCR reaction (Mak et al., 2008). Target gene amplicons accumulate in the polyacrylamide matrix of the droplet where they are hybridized with fluorescent probes, thus labeling the picoreactors. Labeled picoreactors can subsequently be separated from empty picoreactors and picoreactors not containing the target gene using flow cytometry. The labeling is highly specific to the target gene because a fluorescent signal requires both successful PCR amplification and successful hybridization to the target amplicon. The amplification is done using deoxyuridine instead of deoxythymidine to permit amplicon degradation by uracil-specific excision reagents after the flow cytometric selection.

\section{Flow-Cytometric Validation of the Method}

Six different suspensions of E. coli XL1 and E. coli MC1061 cells were prepared in which the percentage of E. coli XL1 ranged from 0 to $100 \%$ of the total. The genome of E. coli XL1 contains a tetracycline resistance gene, whereas the genome of $E$. coli MC1061 does not. The cells were processed as described above to amplify the genomes and label them based on the presence of the tetracycline resistance gene. When analyzed using a flow cytometer, picoreactors carrying the E. coli XL1genome appeared as bright fluorescent events (Figure 6). The less-fluorescent events correspond to empty picoreactors and picoreactors containing the E. coli MC1061 genome. Altogether, 100,000 events were collected for each suspension. The proportion of E. coli XL1 to MC1061 cells remained similar between the initial cell suspension and the labeled picoreactors. Based on the scatterplots, as little as $0.1 \%$ E. coli XL1 cells in the initial cell suspension (relative to MC1061 cells) could be differentiated using the method. The number of fluorescent events correspond to the percentage of XL1 cells (power equation $R^{2}$-value 0.97; Figure 7). 


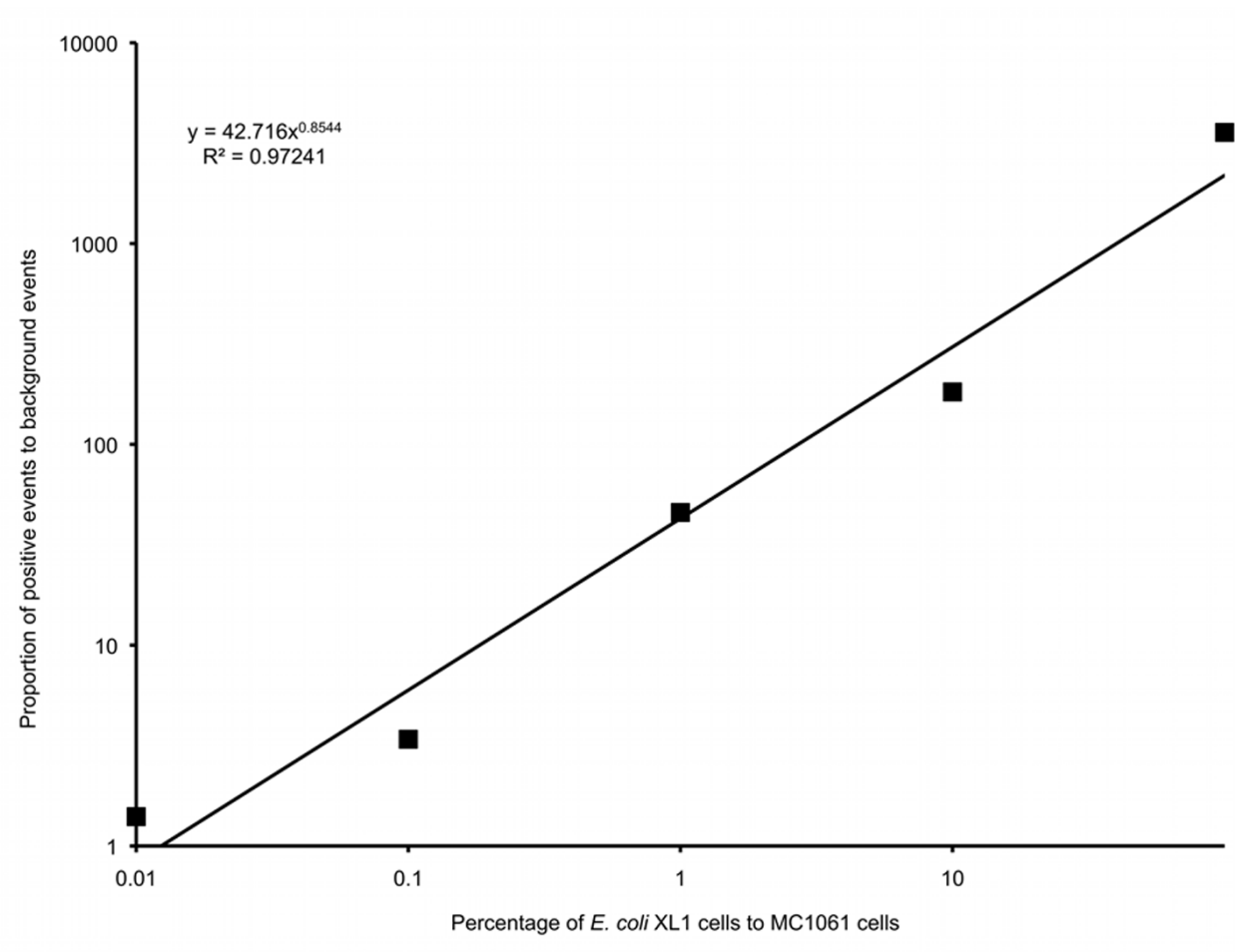

FIGURE 7 | A logarithmic plot of the proportion of false positive background events to positive events in gate P1 versus the percentage of $E$. coli XL1 to $E$. coli MC1061 in the initial cell suspension.

\section{Flow-Cytometric Application of the Method to a Spiked Environmental Sample}

Four different suspensions of E. coli XL1 and diverse microbial cells extracted from marine sediment were prepared where the percentage of $E$. coli XL1 ranged from 0 to $1 \%$ of the total cells. The genome of $E$. coli XL1 contains a tetracycline resistance gene, whereas the sediment microbes do not, according a PCR reaction using isolated sediment DNA as a template (data not shown). The cells were processed as described above to amplify the genomes and label them based on the presence of the tetracycline resistance gene. When analyzed using a flow cytometer, picoreactors carrying the E. coli XL1genome appeared as bright fluorescent (Figure 8). The lessfluorescent events correspond to empty picoreactors and picoreactors containing other genomes. Altogether, 100,000 events were collected for each suspension. The number of false positive background events is higher with an environmental sample than with the mixture of two E. coli strains. Nevertheless, mixtures with 0.1 and $1 \%$ of $E$. coli XL1 exhibited an elevated amount of fluorescent events. With the observed frequency of false positive events, target proportions of $0.01,0.1$ and $1 \%$ could be identified with respective false positive rates of 8 false to 2 true, 6 false to 4 true, and 1 false to 16 true. The false positive background events appear even when a fluorescent probe has not been hybridized to the picoreactors and are most likely autofluorescent cells or mineral particles.

\section{Discussion}

Emulsions are an inexpensive and simple way to divide chemical or enzymatic reactions into millions of parallel reactions. This property of emulsions have been successfully exploited for a number of applications, including pyrosequencing technology, and BEAMing (Margulies et al., 2005; Diehl et al., 2006). Preparing a cell suspension by chemical and mechanical detachment of microbes from biofilm structures and filaments allows an emulsion approach to be used to cast polymer shells on a large quantity of cells without bias from cell morphology (Zengler et al., 2002). Here, polyacrylamide was polymerized on microbial cells in an emulsion to construct a support matrix for the genomic material. This is necessary because some cells are highly resistant to cell lysis, whereas others lyse completely even after a brief enzymatic treatment. The technique presented in this study allows extended incubation times with high concentrations of lytic enzymes because it supports genomic DNA as a discreet package, even after the cell wall and other structures have been completely degraded. Therefore, this method can be used to lyse all cell types, from fragile to highly resistant, in the same reaction. Although the model organism in this study was a Gram-negative bacterium, we expect that the method is equally applicable to different bacterial, archaeal, and eukaryotic cells. Traditionally, the different lysis reaction requirements for different cell types and the risk of complete cell lysis and genome dispersion have posed major difficulties for methods such as CARD-FISH and in situ-PCR, which 


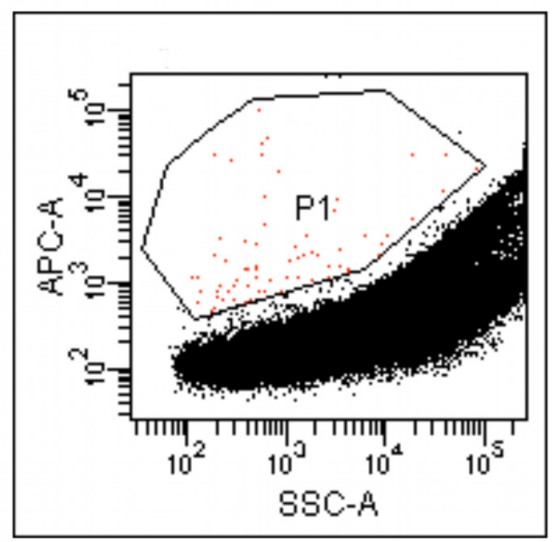

$0 \%$ XL1 cells

67 events in $\mathrm{P} 1$

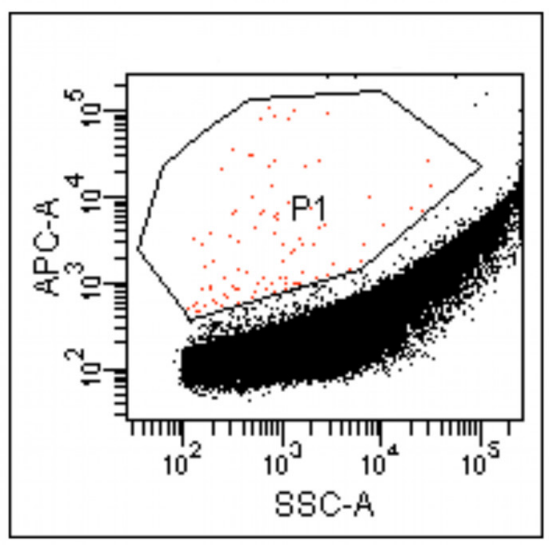

$0.1 \%$ XL1 cells

95 events in $\mathrm{P} 1$

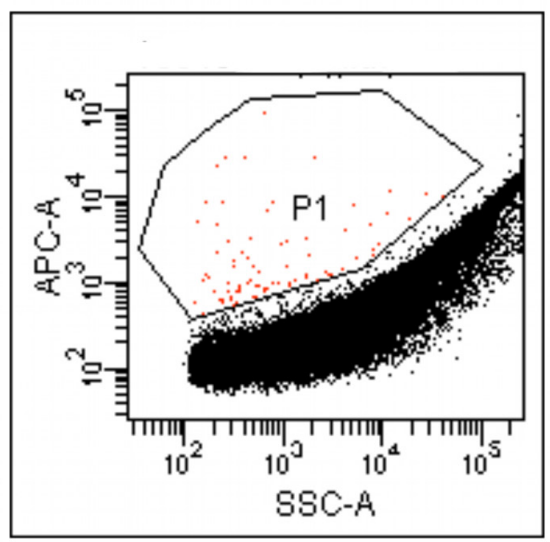

$0.01 \%$ XL1 cells

\section{2 events in $\mathrm{P} 1$}

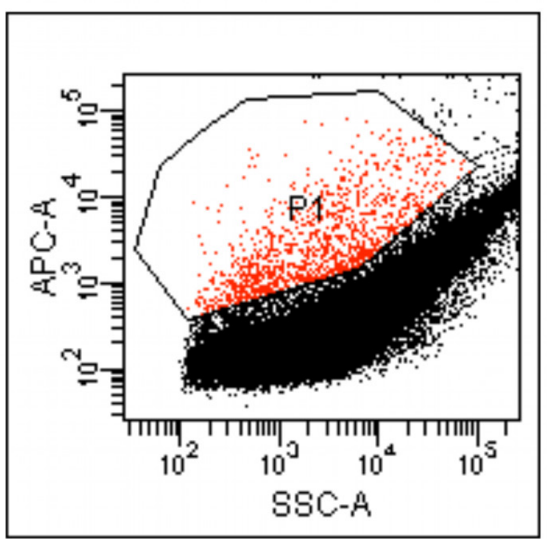

$1 \%$ XL1 cells

1151 events in $\mathrm{P} 1$
FIGURE 8| Flow cytometric results from picoreactors of different suspensions of $E$. coli XL1 and microbes extracted from marine sediment. The $E$. coli $\mathrm{XL1}$ genome contains a single copy of a

tetracycline resistance gene, whereas the sediment microbes do not contain the gene. The genomes are amplified in agarose picoreactors and labeled by emulsion PCR and fluorescent probe hybridization targeting the tetracycline resistance gene. Picoreactors containing the XL1 genome exhibit increased red fluorescence. The parameter SSC-A on the $x$-axis refers to a side-scatter value that correlates with the light-scattering property of the analyzed particles. The parameter APC-A on the $y$-axes refers to the intensity of red fluorescence. Events in the P1 gate are labeled picoreactors containing XL1 genomes and therefore have increased red fluorescence. Altogether, 100,000 events were collected from each suspension. The sediment microbes exhibit a higher rate of false positive events than the mixture of the two E. coli strains (Figure 6), as indicated by the suspension containing no $\mathrm{XL1}$ cells $(0 \%)$. also rely on exposing genomic DNA to enzymes (Hodson et al., 1995; Kubota et al., 2008).

The polyacrylamide support matrix was constructed using a cross-linker ontaining a disulfide bond that can effectively be cleaved in mild reducing conditions, such as in an MDA reaction. Such crosslinker has been used previously to permanently reshape polyacrylamide gels by consequtive reduction, reshaping and oxidation steps (Greytak et al., 2001). This study presents the first demonstration of the use of such material to construct scaffoldings for miniaturized reactors for parallel MDA reactions. Agarose was chosen as the material for the reactor walls because of its sufficient porosity to permit reagent diffusion and its inertness in PCR reactions (Mak et al., 2008). The agarose picoreactors permit the MDA reactions to be performed in an emulsion to avoid any cross-contamination between individual reactors. After the MDA reaction, a second layer of polyacrylamide was prepared using the disulfide bond-containing crosslinker and an $5^{\prime}$-acrydite-modified primer that becomes covalently attached to the polyacrylamide matrix. Acryditemodified primers have previously been used to provide covalent attachment of primers and amplicons to polyacrylamide support matrix in, e.g., polony sequencing technology (Shendure et al., 2005). To our knowledge, covalently bound amplicons have not previously been used as targets for FISH probes. 
The screening procedure is sensitive enough to detect microbial genomes in which the target gene is present in as little $0.1 \%$ of the total initial cell population. The method performs well on complex sediment microbial population spiked with low amounts of E. coli XL1, despite the rate of false positive events that increases with dilution. Until now, such target-gene screening of genomes has been done by testing single amplified genomes on 96-well plates by PCR (Stepanauskas and Sieracki, 2007), an approach that would require screening an average of 10 plates to find one target genome with a target-gene frequency of $0.1 \%$. Clearly, a high-throughput screening procedure such as that described here would be invaluable for studying microbes that are not predominant community members.

Multiple displacement amplification reaction is known to function in nanoliter volumes in an efficiency comparable to a standard reaction volume of microliters (Marcy et al., 2007). Although we could observe DNA amplification by MDA also in picoliter-volume, we cannot currently draw conclusion about the efficiency of the MDA reaction. We envision that in future applications, the MDA-amplified DNA could be reamplified and used for sequencing applications. This would require circumventing the paraformaldehyde fixation of the cells and using a different polymer chemistry that does not cause

\section{References}

Abramoff, M., Magelhaes, P., and Ram, S. (2004). Image processing with imageJ. Biophoton. Internat. 11, 36-42.

Amann, R. I., Ludwig, W., and Schleifer, K. H. (1995). Phylogenetic identification and in-situ detection of individual microbial cells without cultivation. Microbiol. Rev. 59, 143-169.

Chung, K., and Deisseroth, K. (2013). CLARITY for mapping the nervous system. Nat. Meth. 10, 508-513. doi: 10.1038/nmeth.2481

Dean, F. B., Hosono, S., Fang, L., Wu, X., Faruqi, A. F., Bray-Ward, P., et al. (2002). Comprehensive human genome amplification using multiple displacement amplification. Proc. Natl. Acad. Sci. U.S.A. 99, 5261-5266. doi 10.1073/pnas.082089499

De Long, E. F., Wickham, G. S., and Pace, N. R. (1989). Phylogenetic stains ribosoman RNA-based probes for the identification of single cells. Science 243 1360-1363. doi: 10.1126/science.2466341

Diehl, F., Li, M., He, Y. P., Kinzler, K. W., Vogelstein, B., and Dressman, D. (2006). BEAMing: single-molecule PCR on microparticles in water-in-oil emulsions. Nat. Meth. 3, 551-559. doi: 10.1038/nmeth898

Greytak, A. B., Grosberg, A. Y., and Tanaka, T. (2001). Shape imprinting due to variable disulfide bonds in-polyacrylamide gels. J. Chem. Phys. 114, 1055110556. doi: $10.1063 / 1.1369139$

Hodson, R. E., Dustman, W. A., Garg, R. P., and Moran, M. A. (1995). In-situ PCR for visualization of microscale distribution of specific genes and gene-products in prokaryotic communities. Appl. Environ. Microbiol. 61, 4074-4082.

Kallmeyer, J., Smith, D. C., Spivack, A. J., and D'Hondt, S. (2008). New cell extraction procedure applied to deep subsurface sediments. Limnol. Oceanogr. Meth. 6, 236-245. doi: 10.4319/lom.2008.6.236

Keasling, J. D., Palsson, B. O., and Cooper, S. (1991). Cell-cycle-specific F plasmid replication: regulation by cell size control of initiation. J. Bacteriol. 173, 2673-2680.

Kubota, K., Imachi, H., Kawakami, S., Nakamura, K., Harada, H., and Ohashi, A. (2008). Evaluation of enzymatic cell treatments for application of CARD-FISH to methanogens. J. Microbiol. Meth. 72, 54-59. doi: 10.1016/j.mimet.2007.10.006

Mak, W. C., Cheung, K. Y., and Trau, D. (2008). Diffusion controlled and temperature stable microcapsule reaction compartments for
DNA damage such as PEG-acrylates (Chung and Deisseroth, 2013).

In the present state of the technique, the combination of of in situ PCR and FISH to detect low copy genomic targets in rare members of highly diverse cell populations will permit entirely novel experimental possibilities in microbiology and metagenomics.

\section{Author Contributions}

MT and MV designed research; MT performed research; MT analyzed data; MT and MV wrote the paper.

\section{Acknowledgments}

This study was financially supported by the Maj and Tor Nessling Foundation and Academy of Finland. We would like to thank A. Hynninen, A. Karkman, C. Lyra, and L. Pitkänen for their suggestions and remarks, which considerably improved the quality of the manuscript, and M. Molin for his kind assistance with microscopy.

high-throughput microcapsule-PCR. Adv. Funct. Mater. 18, 2930-2937. doi: $10.1002 /$ adfm. 200800388

Marcy, Y., Ishoey, T., Lasken, R. S., Stockwell, T. B., Walenz, B. P., Halpern, A. L., et al. (2007). Nanoliter reactors improve multiple displacement amplification of genomes from single cells. PLoS Genet. 3:1702-1708. doi: 10.1371/journal.pgen.0030155

Margulies, M., Egholm, M., Altman, W. E., Attiya, S., Bader, J. S., Bemben, L. A., et al. (2005). Genome sequencing in microfabricated high-density picolitre reactors. Nature 437, 376-380. doi: 10.1038/nature03959

Shendure, J., Porreca, G. J., Reppas, N. B., Lin, X. X., McCutcheon, J. P., Rosenbaum, A. M., et al. (2005). Accurate multiplex polony sequencing of an evolved bacterial genome. Science 309, 1728-1732. doi: 10.1126/science.1117389

Stepanauskas, R., and Sieracki, M. E. (2007). Matching phylogeny and metabolism in the uncultured marine bacteria, one cell at a time. Proc. Natl. Acad. Sci. U.S.A. 104, 9052-9057. doi: 10.1073/pnas.0700496104

Williams, R., Peisajovich, S. G., Miller, O. J., Magdassi, S., Tawfik, D. S., and Griffiths, A. D. (2006). Amplification of complex gene libraries by emulsion PCR. Nat. Meth. 3, 545-550. doi: 10.1038/nmeth896

Woyke, T., Sczyrba, A., Lee, J., Rinke, C., Tighe, D., Clingenpeel, S., et al. (2011). Decontamination of MDA Reagents for single cell whole genome amplification. PLOS ONE 6:e26161. doi: 10.1371/journal.pone. 0026161

Zengler, K., Toledo, G., Rappe, M., Elkins, J., Mathur, E. J., Short, J. M., et al. (2002). Cultivating the uncultured. Proc. Natl. Acad. Sci. U.S.A. 99, 15681-15686. doi: 10.1073/pnas.252630999

Conflict of Interest Statement: The authors declare that the research was conducted in the absence of any commercial or financial relationships that could be construed as a potential conflict of interest.

Copyright (C) 2015 Tamminen and Virta. This is an open-access article distributed under the terms of the Creative Commons Attribution License (CC BY). The use, distribution or reproduction in other forums is permitted, provided the original author(s) or licensor are credited and that the original publication in this journal is cited, in accordance with accepted academic practice. No use, distribution or reproduction is permitted which does not comply with these terms. 